Revista Electrónica Complutense de Investigación en Educación Musical ISSN-e: 1698-7454

http://dx.doi.org/10.5209/RECIEM.54834

\title{
El sentido de la educación musical en una educación concebida como motor de la economía del conocimiento: una propuesta de marco filosófico ${ }^{1}$
}

\author{
Antonio Fernández-Jiménez ${ }^{2}$; María-Cecilia Jorquera-Jaramillo ${ }^{3}$
}

Recibido: 26 de diciembre de 2016 / Aceptado: 14 de septiembre de 2017

Resumen. Los cambios producidos por la Sociedad y la Economía del Conocimiento están presentes también en las políticas educativas que reducen la importancia de las enseñanzas artísticas y la música en los currículos. En este artículo se revisa la bibliografía sobre la educación musical actual, encontrándose dos enfoques contrapuestos: económico y ciudadano. El segundo se incluye en una educación que pretende desarrollar las múltiples facetas del ser humano para participar en la sociedad actual. La música contribuye a este enfoque ciudadano, pero para ello se necesita elaborar un pensamiento filosófico sobre educación musical capaz de fundamentarla y que responda a temas relevantes, generando conocimiento y acción a través de la música para producir cambios.

Palabras claves: Educación musical; enfoques educativos; filosofía de la educación musical; sociedad del conocimiento; economía del conocimiento.

\section{[en] The sense of music education in education conceived as an engine of the knowledge economy: a philosophical framework proposal}

\begin{abstract}
Changes produced by the Knowledge-based Society and Economy have an influence on educational policies, attaching less importance to arts and music education within the curriculum. This article offers a literature review on present music education, where two opposite perspectives were found in: economic and social. The latter educational perspective aims the development of multiple human aspects in order to participate in current society. Music contributes to this goal, generating knowledge and action by means of it. To achieve this, philosophical thinking on music education, able to give fundament to music education and to respond to relevant topics for current education, should be elaborated. Keywords: Music education; educational perspectives; philosophy of music education; knowledge-based society; knowledge-based economy.
\end{abstract}

Sumario. 1. Introducción. 2. El dilema de la educación musical en la actualidad. 3. Fundamentos filosóficos para la educación musical. 4. Propuestas para asignar sentido a la educación musical. 5. Referencias bibliográficas.

1 Este artículo es parte del proyecto de investigación I+D EDU2014-58066-P sobre El impacto de la educación musical en la sociedad y en la economía del conocimiento financiado por el Ministerio de Economía y Competitividad del Gobierno de España.

2 Universidad de Granada (España) antoniferjim@ugr.es

3 Universidad de Sevilla (España) mcjorquera@us.es 
Cómo citar: Fernández-Jiménez, A.; Jorquera-Jaramillo, M.C. (2017). El sentido de la educación musical en una educación concebida como motor de la economía del conocimiento: una propuesta de marco filosófico, en Revista Electrónica Complutense de Investigación en Educación Musical, 14, 95-107.

\section{Introducción}

Vivimos en una etapa de profundos cambios, en transición hacia una Sociedad y Economía del Conocimiento, debido a los fenómenos migratorios, las nuevas tecnologías y a la globalización. Estos cambios afectan a la sociedad en su conjunto y a los currículos escolares en particular (Avalos, 1999; Marcelo, 2001). En educación musical, el cambio más notable lo vemos en el paso de la educación musical como asignatura obligatoria en las anteriores Leyes Orgánicas, la 1/1990, de 3 de octubre, de Ordenación General del Sistema Educativo (LOGSE) y en la 2/2006, de 3 de mayo, de Educación (LOE), a ser una materia optativa condicionada a que cada centro educativo la oferte o no, según la actual Ley Orgánica 8/2013, de 9 de diciembre, para la mejora de la calidad educativa (LOMCE). La música casi siempre ha tenido que justificar su presencia dentro de los currículos nacionales, pero el caso de España en la actualidad, no tiene precedentes desde que se incorporó a nuestro sistema educativo a principios de los noventa.

A fin de estudiar qué está pasando con la música en la escuela, en tiempos recientes han aparecido diferentes iniciativas, como es el caso de la investigación dirigida por Vilar (2013) a nivel de Primaria en el ámbito catalán. Casals, por su parte, diseña y pone a prueba un proyecto para incrementar el impacto de la educación musical escolar a nivel de centro (Casals, 2013). Existen también otros proyectos que se centran en la integración de la música con otras áreas del currículo desde la base de una acción educativa holística (Bordons y Casals, 2012). Asimismo, el proyecto I+D EDU2014-58066-P actualmente en fase de realización sobre El Impacto de la Educación Musical en la Sociedad y en la Economía del Conocimiento desde el que se produce este artículo se propone igualmente hacer un estudio retrospectivo y prospectivo del lugar que ha jugado, juega y puede tener la música dentro del sistema educativo obligatorio en España.

A nivel internacional encontramos proyectos con este mismo propósito como es el caso de la red Comenius Menet ${ }^{4}$, que estuvo activa del 2006 al 2009. Su propósito era documentar buenas prácticas docentes en educación musical escolar a nivel europeo. También encontramos una iniciativa de investigadores y profesionales de la educación musical del Reino Unido, denominada Inspire-music: Improving music learning together ${ }^{5}$. Ésta busca divulgar buenas prácticas docentes, ofrecer apoyo al profesorado de música de ese país y reflexionar sobre las implicaciones de la educación musical escolar. Cabe mencionar igualmente a Winner, Goldstein y Vicent-Lancrin (2014), quienes hicieron un informe para la Organización para la Cooperación y el Desarrollo Económico (OCDE) sobre el impacto de la educación artística en educación, consistente en hacer una revisión de la literatura al respecto, centrándose en los estudios cuantitativos y entendiendo por «educación», educación para la economía del conocimiento.

Music Education Network.

El enlace a la web del proyecto es: http://www.inspire-music.org/ 


\section{El dilema de la educación musical en la actualidad}

El estudio de la documentación de las recientes políticas de reforma a nivel global muestra que la música tiene cada vez un menor peso en el currículo escolar obligatorio en favor de las denominadas asignaturas STEM (Science, Technology, Engineering and Mathematics), pues son consideradas por diversas instituciones económicas (OCDE, Banco Mundial, Fondo Monetario Internacional) y políticas (la Unión Europea) transnacionales, como los conocimientos imprescindibles que una persona tiene que adquirir para poder hacer frente a los desafíos que presenta la Economía del Conocimiento (Aróstegui, Louro y Teixeira, 2015; Rusinek y Aróstegui, 2015). Además, esta visión refleja la creencia en que la educación debe orientarse hacia la futura ocupación laboral de las personas, excluyendo aquellas actividades, como la música, que llegaron a calificarse en su momento como "que distraen" por el ministro del ramo.

En consonancia con este modelo de currículo se desarrolla cada año el Programa Internacional para la Evaluación de Estudiantes (PISA) que presenta datos sobre los resultados escolares de los alumnos de cada país participante de acuerdo a un modelo muy concreto de evaluación, estandarizado y basado exclusivamente en indicadores numéricos. En esta evaluación sólo se tienen en cuenta la lectura, las matemáticas y las ciencias, por considerar la agencia de evaluación que la lleva a cabo que son éstas las asignaturas susceptibles de ser evaluadas desde un punto de vista cuantitativo y de predecir el éxito escolar, dejando de lado a otras materias, como es el caso de la música, cuya naturaleza difiere sensiblemente de la de esas pruebas evaluativas (Aróstegui, 2016; Rusinek y Aróstegui, 2015). Para estos y otros autores, la música no se puede evaluar en exclusiva con mediciones cuantificables, pues la naturaleza de la educación musical va más allá de reconocer un intervalo en concreto o la precisión en el ritmo, por ejemplo. Desde esta perspectiva, la música escolar pretende que el niño o niña escuche, perciba, exprese, interprete la música y que, en definitiva, desarrolle su capacidad artística y estética. Este enfoque de educación musical no se puede evaluar desde lo cuantificable, ya que se estaría obviando el contexto y que hace que lo que pueda resultar expresivo para unos no lo sea para otros, y viceversa.

Ese modelo de evaluación basado exclusivamente en lo cuantitativo repercute en el diseño de currículos escolares basados en la adquisición de competencias y una evaluación educativa a través de estándares. Esta idea de currículo viene dado por las prácticas a cargo de la OCDE de aplicar los mismos criterios del ámbito empresarial a la educación, pues ésta se considera como un servicio más dentro del modelo neoliberal (Aróstegui, Louro y Teixeira, 2015). Ante esta tendencia, los autores recién citados se preguntan por qué las reformas de las políticas educativas de estas instituciones reducen cada vez más las enseñanzas artísticas en los currículos escolares cuando ellas mismas demandan personas más creativas e innovadoras para hacer frente a los desafíos actuales de la Sociedad y la Economía del Conocimiento, a lo que responden que la música no sólo se debe enseñar por ser un hecho artístico, sino porque contribuye en el desarrollo de las capacidades cognitivas, emocionales, físicas e intelectuales del niño o niña, por lo que la educación musical sirve para formar ciudadanos con pensamiento crítico que participarán activamente en la sociedad y tendrán que afrontar los numerosos retos que ésta presenta.

Sin embargo, no sólo se debe educar para el ámbito económico, también para el ciudadano (Chapman y Aspin, 2001, citado en Marcelo, 2001), puesto que la edu- 
cación musical puede contribuir a desarrollar el sentido estético y cívico, la capacidad para que niños y niñas aporten su propia opinión y criterio, y fomentar el pensamiento crítico y emancipador. En este sentido, Carrillo (2005) hablaba de que para un desarrollo correcto y sostenible de la sociedad y economía del conocimiento (la cual se caracteriza por moverse no únicamente por los factores tradicionales de producción como eran la materia prima y la energía, sino que toma un peso todavía mayor el conocimiento y la capacidad para generarlo y difundirlo) tiene que existir un equilibrio entre los componentes racional, científico, tecnológico, emocional y cultural. En la Declaración Mundial sobre Educación para Todos (UNESCO, 1990) se recoge que niños, jóvenes y adultos deben de alcanzar y mantener un nivel aceptable de aprendizaje, en el cual no se hace distinción entre aprendizaje económico, científico o cultural, ya que lo que se persigue es satisfacer las necesidades básicas de aprendizaje que tienen las personas. Además, el desarrollo de la educación implica la transmisión, es decir la reconstrucción y enriquecimiento de la herencia cultural de la sociedad (Gimeno y Pérez Gómez, 2000; UNESCO, 2000). Por tanto, la educación aparece como un elemento que debe servir de unión entre los diferentes componentes, es decir, lo racional, científico, tecnológico, emocional y cultural, y no que constituya una separación mayor, pues no se puede obviar el derecho que tienen las personas a adquirir una competencia emprendedora, pero tampoco la de ser competentes cívicamente y a tener conciencia de su legado cultural y artístico (Rusinek y Aróstegui, 2015). La sociedad no puede caminar sólo de la mano de lo económico, sino que necesita también del fomento de valores como la solidaridad, la tolerancia, la igualdad, el respeto y la justicia como base para lograr la cohesión social y poder llevar a cabo una vida en común. La educación es, en suma, la encargada de desarrollar a los estudiantes como personas formadas en sus diferentes facetas, más que como simples trabajadores destinados a cumplir los objetivos marcados por el centro de trabajo y producción (Elliott, 1995).

En un mundo tan globalizado en lo político, en lo económico y en lo cultural no hay por qué escoger entre lo económico y lo ciudadano. Nos encontramos ante un momento en el que hay que aunar ambas perspectivas, hacer un planteamiento crítico y reflexivo de esas demandas (Rusinek y Aróstegui, 2015), en el que se pide formar a ciudadanos para el ámbito económico, pero también en el ámbito ciudadano, a personas con capacidad de escucha, crítica y habilidad para interactuar con los otros en un mundo tan globalizado. Esta necesidad de compatibilización no es nueva, es intrínseca al hecho de vivir en una sociedad democrática con una economía de mercado capitalista y desde hace décadas ha sido estudiada fundamentalmente por la pedagogía crítica (v.g., Apple, 1987; Gimeno y Pérez Gómez, 1994; Fernández Enguita, 1997), si bien los fenómenos de la globalización llevan a que esta compatibilidad entre la formación para la ciudadanía y para la economía sea necesaria hoy más que nunca.

A raíz de lo dicho hasta ahora, cabe concluir que esta disyuntiva entre educar para lo económico o para la totalidad de las facetas del ser humano no es tal, sino que se nos presenta como contrapuesto lo que no lo es. Sin embargo, el actual modelo curricular de la LOMCE (2013) sigue apostando por un modelo educativo basado en competencias y en la evaluación de unos estándares de aprendizaje que nos vuelve a llevar a un paradigma positivista y a un diseño de currículo escolar de tipo técnico-racional (Gimeno y Pérez Gómez, 2000), donde la meta del proceso educativo es conseguir lo estipulado en el planteamiento previo. Mientras tanto, la educación 
musical propone el disfrute con la música mediante actividades de improvisación, representación, composición, arreglo y dirección, y también de escucha y observación, consiguiendo así una experiencia musical plena y activa, en la cual se produce un correcto auto-crecimiento (Elliott, 1995). Schippers (2009) amplía esta visión de la educación musical, siguiendo un enfoque de enseñanza holística, que proporciona más espacio a los estudiantes para que construyan su propio conocimiento musical, porque la naturaleza de la tradición musical no es mantener intacta la herencia, sino enriquecerla acorde a las circunstancias del momento y transmitir el resultado a las generaciones futuras, es decir, reconstruir la cultura y el conocimiento recibidos (Gimeno y Pérez Gómez, 2000). Por tanto, los estudiantes tienen que ser participantes activos de esa dinámica, entendiendo que la experiencia musical no es algo ajeno a los cambios, sino que está en un constante proceso de innovación. Sin embargo, la situación de la enseñanza de la música escolar parece estar fuertemente anclada a la tradición de la enseñanza musical especializada, quedando alejada de muchas esferas de la comunidad educativa, pues parece seguir estando presente esa idea en la sociedad de que la música es algo destinado únicamente para personas dotadas de talento.

Mirando hacia la formación del profesorado de música es posible afirmar que todos los formadores provenimos de una misma matriz, es decir, de la formación especializada que proporcionan los conservatorios de música (Duque y Jorquera, 2013). Esto significa que la idea de música que con más frecuencia se transmite en las aulas, es aquella que procede de la cultura académica, o sea aquella que se asocia, precisamente, a la creencia en el talento como dotación innata (Merriam, 1964; Kingsbury, 1988; Nettl, 1995; Jorquera, 2000). Como profesores de música, si 1levamos a las aulas de la enseñanza obligatoria esta cultura profesional, acompañada por la creencia en que quienes practican música deben contar con talento, estaríamos proponiendo una cultura musical lejana respecto a los intereses y las necesidades de aprendizaje de nuestro alumnado que además sería seleccionadora. Por ello, es indispensable elaborar una cultura profesional que se diferencie de manera evidente de aquella que nos impone la tradición de la formación musical especializada, que sea funcional respecto a la idea de ciudadanía mencionada más arriba y que cuente además con fundamentos sólidos. En síntesis, deberíamos elaborar un modelo de educación musical que cuente con fundamentos filosóficos que nos permitan desarrollar el pensamiento crítico en nuestro alumnado.

\section{Fundamentos filosóficos para la educación musical}

Una práctica educativo-musical poco reflexiva, que no dispone de fundamentos filosóficos explícitos, conduce a resultados educativos que pueden estar en contradicción con las intenciones iniciales. En estos casos los docentes no controlan la planificación de su acción educativa, desconociendo numerosos aspectos de su hacer. Hay ciertas críticas que apuntan a que parte de esa vulnerabilidad de la educación musical se debe a los fallos de la anterior filosofía de la educación musical, basada en el desarrollo del sentido estético, estando relacionado, de alguna manera, con una formación musical dirigida a niños y niñas que ya se encuentran dentro del mundo de la práctica musical. Para comprender de manera contextualizada esta cuestión, toma- 
remos como ejemplo la relación entre prácticas educativas y filosofía de la educación musical en EE. UU. (McCarthy y Goble, 2003).

En ese país, un grupo de educadores musicales elaboró un proyecto (Reimer, 1970/ 1989a), apoyado en las ideas de Meyer (2001, publicado originalmente en 1956), que ejerció una fuerte influencia en el panorama de la disciplina durante más de un decenio. A través de la aproximación que se denominó Educación Estética, se logró generar uniformidad en las enseñanzas musicales de numerosos docentes estadounidenses, logrando así unidad en la profesión. A pesar de que quienes promovían este enfoque pretendían que se incluyera la música popular y otros géneros musicales, el núcleo fuerte de la educación musical estaba centrado en la música docta y se daba importancia fundamental a la escucha de obras. Sin duda, es central formar a un público mediante la escucha de música, pero no debería decidirse a priori qué género musical escuchará ese público, sobre todo, teniendo en cuenta que los numerosos géneros de música popular son referencia fundamental para el alumnado. Se pretendía desarrollar la capacidad de juicio crítico sobre la música, sin ampliarla hacia otras esferas de la vida. Además, el enfoque estético dejaba en segundo plano actividades de producción de música, en las que el alumnado pudiese participar tocando instrumentos, improvisando o creando sus propias composiciones, es decir, como protagonistas de las actividades musicales.

Algo similar ocurrió en España cuando se introdujo la música en el sistema escolar con la LOGSE (1990) y en el que el profesorado de música se posicionó en esta perspectiva de la Educación Estética, ya que la casi totalidad de los docentes formadores de profesorado de educación musical procedían de la cultura profesional de los conservatorios de música (Barrios Manzano, 1999). Si se observa, por ejemplo, el contenido de los libros de texto (Jorquera, 2013), se puede apreciar que aún en años recientes se incluyen numerosos contenidos relacionados con el aprendizaje de la lectoescritura y la teoría musical tradicional. Es decir, se siguen los cánones de las enseñanzas impartidas en los conservatorios. Este modo de entender la educación musical es el que constituye la matriz de la cultura profesional del profesorado de música, aún en la actualidad, independientemente del contexto en que cada uno se desenvuelva (Duque y Jorquera, 2013).

Esta forma de entender la educación musical ha calado durante años en la sociedad española, concebida así esta materia por la administración educativa, políticos e incluso familias como una asignatura destinada a personas dotadas de un cierto talento para la música, situación que se da también en otros países y que, de hecho, ha sido una de las razones que ha influido en la pérdida de importancia de la música en el currículo escolar, por lo menos en Estados Unidos, alegando argumentos como que la educación musical no merece apoyo, porque sólo hay un pequeño grupo de estudiantes talentosos, incluso también ha sido utilizado dicho argumento para reducir inversión para esta asignatura, ya que se decide ahorrar fondos justificando que es una materia accesible sólo para unos pocos. Pero la música es accesible, alcanzable y se puede proponer a todos los estudiantes y debe ir de la mano de una formación de calidad de los maestros de música, pues de ella depende que sepan desarrollar en sus estudiantes la musicalidad de una forma efectiva (Elliott, 1995).

Volviendo, una vez más, a cómo se desarrollaron los fundamentos de la educación musical en EE.UU., David Elliott, discípulo de Reimer (autor del enfoque estético de la disciplina), identificó debilidades y lagunas en este planteamiento, llegando a elaborar una nueva filosofía de la educación musical, que se materializó 
con la publicación de la primera edición de Music Matters (Elliott, 1995), texto que acogió ideas presentes entre los educadores musicales desde un decenio antes. Estableciendo un paralelo con la realidad española, considerando que los planteamientos de Elliott tuvieron una cierta difusión en nuestro territorio, se puede afirmar que se desarrolló una tendencia hacia la comprensión de la educación musical como quehacer práctico o como modelo didáctico práctico (Jorquera, 2010). Sin embargo, quizás no se llegó a una visión que refleje el concepto de educación musical praxiológica (Elliott, 2003) que va más allá del solo hacer práctico o, en nuestro caso, de la sola práctica musical. Elliott, en efecto, recurre al concepto aristotélico de praxis, que no se limita al solo hacer, sino que implica un esfuerzo relacionado con el contexto. La praxis tendría entonces un significado de producción cultural, incluso en contextos escolares.

En el texto en el que el autor profundiza sobre el concepto de educación musical praxiológica (2003), Elliott solicitó a algunos educadores musicales que realizaran un examen crítico del texto de 1995, para identificar las fortalezas, debilidades y lagunas que pudiera haber en sus planteamientos. Este trabajo condujo, en última instancia a la publicación de la segunda edición revisada, en coautoría con Marissa Silverman (Elliott y Silverman, 2015), en el que se incluyen nuevas ideas, como el enfoque crítico, entre otras, en consonancia con los tiempos actuales y con la elaboración teórica que se ha ido haciendo en el ámbito de los encuentros del Grupo MayDay6 y en la revista Action, Criticism, and Theory for Music Education, sobre la que se proporcionará información más adelante y que, a nuestro parecer, aportan ideas interesantes sobre la perspectiva que debe orientar a la filosofía de la educación musical en la actualidad.

\section{Propuestas para asignar sentido a la educación musical}

Ante esta encrucijada en la que se encuentra la educación musical en el currículo escolar obligatorio en España, no se puede pasar por alto la necesidad e importancia de elaborar un planteamiento filosófico en la disciplina que vislumbre el camino que tendría que seguir esta materia. Se trata de elaborar una propuesta de filosofía de la educación musical que dé sentido a la finalidad pretendida por el área, así como de dar respuesta a las necesidades que demanda este tipo de sociedad en la que ya estamos inmersos.

En este movimiento filosófico capaz de mover la educación musical actual deben de participar los propios educadores y educadoras musicales, pues son ellos y ellas quienes conocen, de primera mano, la realidad educativa de las aulas del momento. Deben dirigir este pensamiento hacia la creación de una educación musical basada en una práctica que no sea el fruto exclusivo del hacer práctico, sino que confiera un contexto cultural al mismo, es decir que hay que fomentar el desarrollo de un pensamiento musical en acción. Cabe recordar que una educación musical orientada exclusivamente al hacer práctico, entendido como interpretación o como práctica musical, fácilmente se puede centrar en la sola adquisición de conocimientos téc-

Fundado por iniciativa de Thomas Regelski en 1993. En el enlace http://www.maydaygroup.org/ se encuentra la web de este grupo. Aquí es posible leer información sobre el ideario, la historia, las publicaciones y actividades del mismo. 
nico-musicales. La educación musical no sólo debe dirigirse a hacer música, entendiéndola como la creación de sonidos, melodías, ritmos e interpretación de los mismos; es decir, se está presentando la idea de que a través de la música, también, se genere conocimiento en diversos ámbitos, pues es requerido en el contexto en el que vivimos hoy en día (Elliott y Silverman, 2015). La sociedad actual pretende que los jóvenes adquieran los conocimientos, valores, actitudes, orientaciones morales necesarias para saber tratar con temas de género, inclusión social, medio ambiente, respeto, convivencia y atención hacia las desigualdades, por ejemplo. Con esta dinámica bien instaurada en las clases de música de "hacer música" se está dejando de lado que el alumnado adopte una postura generando pensamiento y conocimiento musical en acción que dé respuesta a las necesidades del contexto social que nos rodea (Woodford, 2005).

El valor pedagógico de la educación musical recae precisamente en proporcionar diferentes caminos para crear significados, yendo más allá de la perspectiva técnica, tecnológica y racional. Se trata de abrirnos al conocimiento del mundo que nos rodea y a los agentes que participan y forman parte de él, esto es, redescubrir la humanidad (van der Schyff, 2015). Ese pensamiento musical fomenta capacidades de reflexión, crítica, predisposición, trabajo y responsabilidad que son imprescindibles para formar a ciudadanos que participen activamente en la sociedad (UNESCO, 2006). Para este cometido, se precisa de un nuevo modelo de maestro de música que desarrolle un bagaje de conocimiento y reflexión en ámbito científico, tecnológico, socio-cultural, pero también sobre una ética y un contexto basado en la práctica; que explore distintas formas de ser, musicalmente hablando, desde diversos puntos de vista. Una perspectiva que establezca relaciones con los temas de la sociedad que rodean la escuela, para conseguir una humanidad más ética que se rija nuevamente por auténticas formas de convivir en la sociedad (van der Schyff, 2015).

Que la educación musical no siempre esté desarrollando esta visión de la educación centrada en los asuntos sociales que rodean la escuela, quizás sea otro argumento por el cual se excluye a esta materia de la formación básica propia de la etapa escolar obligatoria. De alguna manera, no se está dando respuesta a esas necesidades, por lo que es considerada como poco relevante para conseguir ese objetivo de la educación universal de calidad. Una educación que necesita de estudiantes que sean capaces de apreciar un mundo que está lleno de responsabilidades y riesgos en diferentes temas que afectan a la sociedad y que la acción creativa y musical puede ayudar a afrontarlos. Bowman (2009) habla de que la educación musical se encuentra en una situación en la que sus objetivos han ido adoptando una orientación "nihilista", carente de valor y propósito. Es por eso que debe de producirse un cambio, un giro del pensamiento filosófico de la educación musical hacia una materia que dé sentido a la necesidad de su lugar dentro del currículo escolar obligatorio. Una materia que establezca conexiones entre los diversos temas que preocupan a la sociedad y las prácticas educativas que se llevan a cabo en las aulas de música (Bradley, 2012).

Como ya hemos señalado, la aportación a la filosofía de la educación musical de Woodford (2005), muestra cómo en el mundo anglosajón, después de una educación musical de enfoque estético, como la que propone Reimer (1970/1989a y 1989b), se llegó a una orientada a la práctica, poniendo especial atención en los procesos, a partir de los planteamientos de Elliott (1995). En particular, apoyándose en el pensamiento de Dewey, Woodford demuestra que una educación musical centrada exclusivamente en el quehacer práctico musical desconectado de la realidad social no deja 
espacio a la elaboración de pensamiento sobre la música, ni al debate y la reflexión sobre las cuestiones relevantes que se plantean en la sociedad actual: las desigualdades, el multiculturalismo, la ciudadanía, el pensamiento democrático, la necesidad de cambio, las cuestiones de género, el estado del planeta, etc.

Si observamos las características del quehacer musical en las aulas escolares, fácilmente veremos que las enseñanzas aún hoy en gran parte se centran en cuestiones técnicas (Jorquera, 2013) que difícilmente permiten establecer relaciones con otras disciplinas o que se puedan abordar tratando cuestiones relevantes como las mencionadas poco más arriba. Habitualmente, el profesorado dedica gran atención al quehacer práctico, que en numerosas ocasiones figura como una actividad descontextualizada, utilizada por los centros para fiestas o eventos a lo largo del curso. En estos casos, aun proporcionando al alumnado ocasiones insustituibles de práctica y vivencia musical, si no logramos ir más allá, produciendo conocimiento y pensamiento sobre la música, podría transformarse en una actividad ornamental y hasta prescindible. Por tanto, es indispensable un cambio de visión por parte del profesorado, que amplíe las concepciones acerca de lo que debe suceder en las aulas y dando lugar a una enseñanza que preste atención a las temáticas mencionadas anteriormente, a través de la música, obviamente.

Son temas que en la literatura en lengua española todavía tienen escasa presencia, pero ya cuentan con numerosas aportaciones en lengua inglesa. El grupo MayDay ha desarrollado un interesante debate en torno a estas cuestiones, dando lugar a diferentes publicaciones, entre las que destaca la revista Action, Criticism, and Theory for Music Education antes mencionada.

Entre las cuestiones tratadas en los últimos años citamos, entre otros muchos títulos, los números monográficos sobre las jornadas llevadas a cabo por el grupo MayDay. Por ejemplo, el artículo de Bates (2013) que presenta algunas de las intervenciones que tuvieron lugar en las jornadas de 2011, en que se trató la cuestión de un posible final de la educación musical, teniendo en cuenta que incluso en otras disciplinas se asumía una perspectiva análogamente fatalista. Otro monográfico, desarrollado a partir del debate que las mencionadas jornadas generaron, es el que presenta Elliott (2013) planteando la necesidad de renovar no sólo los fines de la educación musical, sino también el ideario del propio Grupo MayDay. El artículo de Goble (2013), en línea con el debate anteriormente mencionado, se cuestiona sobre la adecuación de los repertorios respecto a lo que se trabaja en las aulas, evidenciando la importancia de no limitar el estudio de los mismos a las cuestiones técnicas, sino de incluir el estudio de los contextos y significados de esos repertorios.

Vale igualmente la pena mencionar el artículo de Lamb (2014), que destaca cómo las mujeres han sido casi totalmente excluidas de los estudios sociológicos sobre educación musical, en particular en los dos períodos que la autora examina.

Bradley (2015), al realizar una recensión de la nueva edición de Music Matters (Elliott y Silverman, 2015), toca cuestiones relacionadas con el multiculturalismo, como es el concepto de cultura "híbrida", que implica una cultura sujeta a cambios debido a la migración global, abandonando la idea de una cultura estática. Para la autora, el esencialismo musical de una tradición cultural estática carece de sentido en un mundo en el que cambia la tradición según las necesidades del momento para seguir manteniendo el interés del público. Desde esta perspectiva, se aboga por un currículo musical práctico que pretenda una reflexión más profunda y consciente de estructuras de poder que influyen a la hora de hacer y escuchar música bajo las 
diferentes culturas musicales y sus consecuencias para el desarrollo de la sociedad. Así, se presenta la oportunidad de examinar y actuar positivamente para eliminar los racismos inconscientes que todavía existen en la tradición cultural y musical que se trabaja en las aulas de educación musical. También Sagar y Hebert (2015) se refieren al multiculturalismo en el currículum, tomando como referencia un proyecto específico realizado en Guyana.

Un texto de reciente publicación es The Oxford Handbook of Social Justice in Music Education (Benedict, Schmidt, Spruce y Woodford, 2015), que trata en profundidad temas de gran relevancia relacionados con las cuestiones mencionadas en este apartado. Son sólo algunos ejemplos que pueden ser estimulantes para el profesorado y que podrían conducir a la elaboración de pensamiento teórico que sustente las actuaciones de aula en nuestro contexto, permitiendo a la educación musical seguir las sugerencias de documentos como la Declaración Mundial sobre Educación para Todos (UNESCO, 1990) o el referido a un Futuro Sostenible para Todos (UNESCO, 2016).

En suma, se puede apreciar cómo fuera de las fronteras de nuestro país se habla de una filosofía de la educación musical sobre la que sustentar el desarrollo de esta disciplina. Sin embargo, no encontramos bibliografía en español acerca de un pensamiento filosófico que guíe la educación musical hacia un nuevo modelo que vaya más allá del quehacer práctico. Con esto nos referimos a un planteamiento de educación musical en el que el alumnado interprete, cree y escuche música, pero también trabaje y reflexione sobre temas y aspectos que conciernen de forma activa a la sociedad actual. Es esta carencia la que sustenta la necesidad de elaborar una filosofía de la educación musical que pueda ofrecer un sentido para que esta materia quede dentro del currículo escolar obligatorio de manera permanente en el tiempo.

$Y$ es que estamos haciendo referencia al continuo peligro de que la música pueda desaparecer por completo del currículo escolar obligatorio. Esta situación ha producido una tendencia constante de defensa de la educación musical escolar, tema que siempre ha estado presente, tal y como ya comentaba Bresler (2003). Este hecho puede deberse a que la mayoría de los argumentos en defensa de la educación musical que se daban y se dan, no provienen de investigaciones científicas. Sin embargo, la educación no puede basarse en ocurrencias de músicos y educadores probablemente bienintencionados pero que no dan respuesta a las demandas y necesidades de la escuela en su totalidad y, ni mucho menos a las reformas curriculares actuales, que ponen su punto de mira en formar a personas con los conocimientos necesarios para hacer frente a los desafíos que presenta la Sociedad y la Economía del Conocimiento. Por eso, desde aquí nos hacemos eco de la necesidad de trabajar en la elaboración de una filosofía de la educación musical que dé respuesta, por un lado, a las necesidades que esta Sociedad y Economía del Conocimiento demanda y, por otro, al desarrollo del ámbito ciudadano, que es realmente necesario para una convivencia democrática, participativa e igualitaria de la humanidad en un mundo tan globalizado como el de la actualidad.

Para finalizar, de lo dicho en el artículo se desprende que las reformas educativas han ido por un lado yla educación musical por otro. Ese otro camino de la educación musical, hasta la actualidad, no ha contemplado de manera clara los fundamentos de la misma, es decir,laelaboracióndepensamientofilosóficocapazdejustificarydeproponerreferentes sólidos para lo que se hace en las aulas escolares. Por tanto, dejamos constancia de la necesidad e importancia de poner en contexto las prácticas musicales, pues se 
trata de la llave para vincular los problemas del sistema escolar con la educación musical y que esta última esté presente dentro del currículo escolar con pleno derecho y en posición de paridad respectoa otras asignaturas. Un camino posible para la educación musical, que la colocaría en un contexto de riqueza educativa en línea con muchas de las ideas sugeridas por la pedagogía crítica.

\section{Referencias bibliográficas}

Apple, M.W. (1987). Educación y poder. Barcelona: Paidós/M.E.C.

Aróstegui, J. L., Louro, A. L. y Teixeira, Z. L. (2015). Las políticas educativas de reforma y su impacto en la Educación Musical Escolar. De dónde venimos y hacia dónde podemos ir. Revista da ABEM, 23(35), 24-34.

Aróstegui, J. L. (2016). Exploring the global decline of music education. Arts Education Policy Review, 117(2), 96-103.

Avalos, I. (1999). La sociedad del conocimiento. Revista SIC, 617, 295-297.

Barrios Manzano, M. P. (1999). La formación del profesorado universitario de música didáctica de la expresión musical: características y problemática. Revista Electrónica Interuniversitaria de Formación del Profesorado, 2(1), 605-609.

Bates, V. C. (2013). MayDay Colloquium 23: The End(s) of Music Education? A Call for Re-Visioning. ACT Action, Criticism, and Theory for Music Education, 12(1), 1-6. Disponible en http://act.maydaygroup.org/articles/BatesA12_1.pdf

Benedict, C., Schmidt, P., Spruce, G., \& Woodford, P. (Eds.). (2015). The Oxford Handbook of Social Justice in Music Education. New York: Oxford University Press.

Bordons, G. y Casals, A. (2012). Poesía, música i escola: Un triangle sonor. Temps d'Educació, 42(1), 101-124.

Bowman, W. (2009). La educación musical en tiempos nihilistas. En Lines, D. K. (Comp.), La educación musical para el Nuevo milenio. Madrid: Ediciones Morata.

Bradley, D. (2012). Good for what, good for whom?: Decolonizing Music Education Philosophies. En W. D. Bowman y A. L. Frega (Ed.), The Oxford Handbook of Philosophy in Music Education. New York: Oxford University Press.

Bradley, D. (2015). The Dynamics of Multiculturalism in Music Matters: A Philosophy of Music Education. ACT Action, Criticism, and Theory for Music Education, 14(3), 10-36. Disponible en http://act.maydaygroup.org/articles/Bradley14_3.pdf

Bresler, L. (2003). The power of music and education in the 21st century: opening up new directions. En S. Leong (Ed.), Musicianship in the 21st Century. Sidney: Australian Music Centre.

Carrillo, J. (2005). ¿Qué es la Economía del Conocimiento? Transferencia, 18(69), 2-3.

Casals, A. (2013). Aplicación de concepto de 'experiencia crítica' al análisis de innovaciones educativas. Tendencias Pedagógicas, 21, 79-97.

Duque, J. y Jorquera, M. C. (2013). Identidades profesionales en educación musical. Diversidad de orígenes para un mismo escenario de práctica. En Re-conceptualizing the Professional Identity of European Teacher: Sharing Experiences (pp. 243-256). JOUR.

Elliott, D. J. (1995). Music Matters: A New Philosophy of Music Education. New York: Oxford University Press.

Elliott, D. J. (Ed.). (2003). Praxial Music Education: Reflections and Dialogues. New York: Oxford University Press. 
Elliott, D. J. (2013). MayDay Colloquium 24: The Aims of Music Education. ACT Action, Criticism, and Theory for Music Education, 12(2), 1-9. Disponible en http://act.maydaygroup.org/articles/Elliott12_2.pdf

Elliott, D. J. \& Silverman, M. (2015). Music Matters: A Philosophy of Music Education. New York: Oxford University Press.

Fernández Enguita, M. (1997). La escuela a examen. Madrid: Pirámide.

Gimeno, J. y Pérez Gómez, Á. I. (1994). Comprender y transformar la enseñanza. Madrid: Morata.

Gimeno, J. y Pérez Gómez, Á. I. (2000). Comprender y transformar la enseñanza. Madrid: Morata.

Goble, J. S. (2013). The Promise and Practice of Pragmatism-Based Music Education in Democratic Societies. ACT Action, Criticism, and Theory for Music Education, 12(2), 8-19. Disponible en http://act.maydaygroup.org/articles/Goble12_2.pdf

Jorquera, M. C. (2000). Educación musical en la sociedad contemporánea. LEEME Revista de la Lista Electrónica Europea de Música en la Educación, 6, 1-14. Disponible en http:// musica.rediris.es/leeme/revista/jorquera00.pdf

Jorquera, M. C. (2010). Modelos didácticos en la enseñanza musical: el caso de la escuela española. Revista Musical Chilena, 64(211), 52-74. Disponible en http://www.scielo.cl/ pdf/rmusic/v64n214/art06.pdf

Jorquera, M. C. (2013). Libros de texto como textos visibles: un estudio piloto. En C. et al. Poblete Lagos (Ed.), Actas $9^{a}$ Conferencia Latinoamericana y $2^{a}$ Panamericana de la Sociedad Internacional de Educación Musical, ISME. 3 al 6 de Septiembre de 2013, Santiago, Chile (pp. 610-620).

Kingsbury, H. (1988). Music, Talent, and Performance. A Conservatory Cultural System. Philadelphia: Temple University Press.

Lamb, R. (2014). Where are the women? And other questions, asked within an historical analysis of sociology of music education research publications: Being a self-reflective ethnographic path. ACT Action, Criticism, and Theory for Music Education, 13(1), 188222. Disponible en http://act.maydaygroup.org/articles/Lamb13_1.pdf

Ley Orgánica 1/1990, de 3 de octubre, de Ordenación General del Sistema Educativo (LOG$\mathrm{SE})$.

Ley Orgánica 2/2006, de 3 de mayo, de Educación (LOE).

Ley Orgánica 8/2013, de 9 de diciembre, para la mejora de la calidad educativa (LOMCE).

Marcelo, C. (2001). Aprender a enseñar para la Sociedad del Conocimiento. Revista Complutense de Educación, 12(2), 531-593.

McCarthy, M., \& Goble, J. S. (2003). The Praxial Philosophy in Historical Perspective. In Praxial Music Education: Reflections and Dialogues (pp. 19-51). Cary, US: Oxford University Press.

Merriam, A. P. (1964). The Anthropology of Music. Evanston, Illinois: Northwestern University Press.

Meyer, L. (2001). La emoción y el significado en la música. Madrid: Alianza.

Nettl, B. (1995). Heartland Excursions: Ethnomusicological Reflections on Schools of Music. Chicago, Urbana: University of Illinois Press.

Reimer, B. (1970). A Philosophy of Music Education. Englewood Cliffs, NJ: Prentice-Hall.

Reimer, B. (1989a). A Philosophy of Music Education. Englewood Cliffs, NJ: Prentice-Hall.

Reimer, B. (1989b). Music education as aesthetic education: Toward the future. Music Educators Journal, 75(7), 26-32. Disponible en http://www.jstor.org/stable/3400308?seq=1\#page_scan_tab_contents 
Rusinek, G. \& Aróstegui, J. L. (2015). Educational Policy Reforms and the Politics of Music Teacher Education. En Benedict, C., Schmidt, P., Spruce, G., \& Woodford, P. (Eds.), The Oxford Handbook of Social Justice in Music Education. New York: Oxford University Press.

Sagar, R., \& Hebert, D. G. (2015). Research-Based Curriculum Design for Multicultural School Music: Reflections on a National Project in Guyana. ACT Action, Criticism, and Theory for Music Education, 14(2), 145-173. Disponible en http://act.maydaygroup.org/ articles/SagarHebert14_2.pdf

Schippers, H. (2009). Facing the music: Shaping music education from a global perspective. New York: Oxford University Press.

UNESCO (1990). Declaración Mundial sobre Educación para todos y Marco de Acción para satisfacer las Necesidades Básicas de Aprendizaje. In Conferencia Mundial sobre Educación para Todos (pp. 1-42). Disponible en http://unesdoc.unesco.org/images/0012/001275/127583s.pdf

UNESCO (2000). Declaración Mundial sobre Educación para Todos. En UNESCO, Marco de Acción de Dakar. Educación para Todos: cumplir nuestros compromisos comunes. Francia: UNESCO.

UNESCO (2006). Hoja de Ruta para la Educación Artística. Conferencia Mundial sobre la Educación Artística: construir capacidades creativas para el siglo XXI. Lisboa: UNESCO.

UNESCO. (2016). Education for people and planet: Creating sustainable futures for all. Paris. Disponible en http://unesdoc.unesco.org/images/0024/002457/245752e.pdf

van der Schyff (2015). Praxial music education and the ontological perspective: An enactivist response to Music Matters 2. ACT Action, Criticism, and Theory for Music Education, 14(3), 75-105. Disponible en http://act.maydaygroup.org/articles/vanderSchyff14_3.pdf

Vilar, M. (2013). Quinze anys d'educació musical a l'escola primària. Informe de treball per a l'ICE-UAB. Disponible en http://grupsderecerca.uab.cat/musicaieducacio/content/ documents-labast

Winner, E., Goldstein, T. R. y Vicent-Lancrin, S. (2014). ¿El arte por el arte? La influencia de la educación artística. París: OCDE.

Woodford, P. (2005). Democracy and Music Education: Liberalism, Ethics, and the Politics of Practice. Bloomington and Indianapolis: Indiana University Press. 